Jeder medizinische Zwischenfall ist einer zu viel - und eine grosse Belastung sowohl für den Patienten als auch für den Arzt. Wird nach einer Behandlung ein Fehler vermutet, stellt sich die Frage nach den weiteren Schritten. Da jedem von uns ein Fehler passieren kann, ist die vorgängige Auseinandersetzung mit diesem Thema wichtig, um umsichtig vorzugehen. Der FMH-Rechtsdienst kennt die Relevanz des Themas für unsere Mitglieder und stellt ihnen als Dienstleistung die wichtigsten Informationen in einem überarbeiteten Merkblatt zur Verfügung.

Dr. med. Jürg Schlup, Präsident der FMH

\title{
Orientierungshilfe im Umgang mit medizinischen Zwischenfällen
}

\begin{abstract}
Ärztinnen und Ärzte sind von Berufs wegen zu Sorgfalt verpflichtet. Sie üben ihre Tätigkeit unter Einhaltung von hohen Qualitätsstandards aus. Dennoch können ihnen auch Fehler unterlaufen. Doch was ist zu tun nach einem medizinischen Zwischenfall?

Tritt ein medizinischer Zwischenfall ein, lässt sich der weitere Ablauf vereinfacht in drei Phasen unterteilen:

Entscheidend ist die erste Phase unmittelbar nach dem Vorfall - die Zeit läuft. Trotz der anfänglichen Verwirrung müssen zunächst der Patient sorgfältig weiterbehandelt und die betroffenen Ärztinnen betreut werden. Letztere gelten nach einem Zwischenfall auch als Opfer, sogenannte second victims. Ihre Betreuung ist wichtig, weil sie sonst Gefahr laufen, erneut Fehler zu begehen. Zudem sind Massnahmen für eine möglichst lückenlose Rekonstruktion des Sachverhalts umzusetzen. Dazu gehören beispielsweise das Nachführen der Krankengeschichte des Patienten oder das Aufbewahren allfälliger Beweismittel. Am besten erstellt jede beteiligte Person ein Gedächtnisprotokoll - andernfalls können die Erinnerungen bis zur Aufnahme eines Verfahrens verblassen. Darüber hinaus bildet eine transparente Kommunikation nach innen und nach aussen eine wichtige Grundlage für das weitere Vorgehen.
\end{abstract}

\section{Der FMH-Rechtsdienst informiert betreffend den Umgang mit medizinischen Zwischenfällen.}

In der zweiten Phase wird angestrebt, Schadenersatz- und Genugtuungsfragen aussergerichtlich zu regeln. Diese Verhandlungen übernehmen die Haftpflichtversicherung für den Arzt und die Anwältin für den Patienten. Für die medizinische Aufarbeitung des Geschehens werden Gutachten veranlasst. Je nachdem wer sie in Auftrag gibt, handelt es sich um Parteigutachten, Gerichtsgutachten oder aussergerichtliche Gutachten. Im Prozess dienen sie als Beweismittel.
Gelingt es den Parteien nicht, eine Lösung zu finden, kommt es in einer dritten Phase zum Zivilprozess um Schadenersatz- und/oder Genugtuungsforderungen. Der Patient muss den Behandlungsfehler, den Schaden und den dazugehörigen Zusammenhang beweisen, die Ärztin muss darlegen, dass sie den Patienten rechtsgenügend aufklärte und dieser in die Behandlung einwilligte. Ein Strafverfahren wird immer dann eingeleitet, wenn der Staatsanwalt den Fall von Amtes wegen untersuchen muss, oder wenn der Patient einen Strafantrag stellt.

\section{Nach einem Behandlungsfehler lässt sich der weitere Ablauf in drei Phasen unterteilen.}

Haftpflichtprozess und Strafverfahren unterscheiden sich grundlegend. In beiden Verfahren können dennoch Zeugen einvernommen werden, die an der Behandlung zwar nicht direkt beteiligt waren, aber trotzdem Aussagen dazu machen können. Wie die Parteien selbst unterliegen sie gewissen Rechten und Pflichten. Sie sind verpflichtet, auf eine Vorladung hin in jedem Fall zu erscheinen, haben in gewissen Fällen aber das Recht, die Aussage zu verweigern. Das Zivilverfahren kann entweder jederzeit durch einen Vergleich abgeschlossen oder durch alle Instanzen bis zum Bundesgericht durchlaufen werden. Im Strafverfahren gibt es verschiedene Möglichkeiten der Beendigung.

Ein Merkblatt mit Informationen über den Umgang mit Behandlungsfehlern finden Sie unter www.fmh.ch $\rightarrow$ Services $\rightarrow$ Recht $\rightarrow$ Praktische Tipps. Als weitere Dienstleistungen können Sie Anfragen zu dieser Thematik an den Rechtsdienst richten oder Vorträge buchen.

Ursina Pally Hofmann, Dr. iur., Rechtsanwältin, stv. Leiterin Rechtsdienst 